\title{
La construcción de la introducción y la conclusión del género "ponencia" en el marco de un taller de escritura académica
}

\section{Writing the Introduction and the Conclusion Sections of a Conference Paper in an Academic Writing Workshop}

\author{
Manuela Álvarez Álvarez ${ }^{1}$ \\ manuela.alvarez@deusto.es \\ Erlantz Velasco Luzuriaga \\ erlantz.velasco@deusto.es \\ Mari Mar Bolllos Pereira \\ mboillosp@gmail.com \\ Universidad de Deusto, España
}

\section{Resumen:}

Este trabajo se centra en el estudio de las secciones introducción y conclusiones de una ponencia producida por estudiantes de doctorado españoles en el contexto de un taller de escritura académica. Su objetivo es analizar la construcción retórica de ambas secciones, a través del modelo teórico Swalesiano basado en el análisis de movimientos, pasos y subpasos. En el caso de las introducciones se toma la propuesta de Carbonell-Olivares, Gil-Salom y Soler-Monreal (2009), mientras que para la sección de conclusiones se emplea el modelo de Yag y Alison (2003). Los resultados de esta investigación en el caso de las introducciones refrendan los datos de

\begin{abstract}
:
This paper studies introduction and conclusion sections of papers produced by Spanish PhD Students in an academic writing workshop. Based on Swales's CARS model, the aim is to analyze the movements, steps, and substeps accomplished in the construction of the introduction (Carbonell-Olivares, Gil-Salom, y SolerMonreal, 2009) and concluding sections (Yag y Alison, 2003). With regard to introductions, results of the study confirm, in line with previous studies, that movement $1(\mathrm{M} 1)$ and $3(\mathrm{M} 3)$ are considered compulsory by Spanish writers. Moreover, participants consider more important to establish the topic of the research than highlighting
\end{abstract}

1 Dirección para correspondencia (correspondence address):

Manuela Álvarez Álvarez. Dpto. de Lenguas Modernas y Estudios Vascos. Facultad de Ciencias Sociales y Humanas. Universidad de Deusto. Avda. Universidades, 24. 48007 Bilbao (España). 
La construcción de la introducción y la conclusión del género "ponencia" en el marco de un taller de escritura académica

Manuela Álvarez Álvarez, Erlantz Velasco luzuriaga y Mari Mar Boillos Pereira

otros estudios donde los movimientos 1 (M1) y 3 (M3) se consolidan como movimientos obligatorios en trabajos de escritores españoles; pero, además, se revela que el establecimiento de la temática tiene un mayor despliegue informativo que la presentación de la propia investigación lo que indica una menor atención del escritor a la originalidad e importancia de su trabajo. En el caso de las conclusiones se observa una mayor integración al modelo prototípico de los tres movimientos establecidos para dicha sección (M1-M2-M3) y se manifiesta una importante conciencia del escritor por el despliegue de información relativa al resumen y valoración de los resultados (M1 y M2).

\section{Palabras clave:}

Escritura académica; análisis de género; ponencia; sección de introducción; sección de conclusión. the significance and originality of the work. In the case of the conclusions, there is a presence of the three prototypical sections (M1-M2-M3). Nevertheless, the main focus in on the summary (M1) and the evaluation of the results (M2).

\section{Résumé:}

Ce travail porte sur l'étude des sections introduction et conclusions d'une communication produite par des étudiants de doctorat espagnols dans le contexte d'un atelier d'écriture académique. L'objectif est d'analyser la construction théorique des deux sections, à travers le modèle théorique Swale basé sur l'analyse de mouvements pas et souspas. Dans le cas des introductions nous reprenons la proposition de Carbonell-Olivares, Gil-Salom et Soler Monréal (2009), alors que pour la section des conclusions nous retenons le modèle de Yag et Alison (2003). Les résultats de cette recherche, dans le cas des introductions, reprennent les données d'autres études où les mouvements 1 (M1) et 3 (M3) se consolident comme des mouvements obligatoires dans les travaux d'écrivains espagnols. De plus, on relève que l'établissement de la thématique présente un déploiement informatique plus important que la présentation de la recherche en elle-même, ce qui indique une moindre attention de l'écrivain envers l'originalité et l'importance de son travail. Dans le cas des conclusions, nous pouvons observer une plus grande intégration au modèle prototypique des trois mouvements établis pour cette section (M1-M2M3) et il se manifeste une grande conscience de l'écrivain envers le déploiement de I'information relative au résumé et à la valorisation des résultats (M1 et M2)

\section{Mots clés:}

Écriture académique; analyse de genre; communication; section d'introduction; section de conclusion.

Fecha de recepción: 9-9-2015

Fecha de aceptación: 26-5-2016 


\section{Introducción}

Las competencias lingüístico-comunicativas, por su incidencia en la incorporación e integración de los estudiantes en el área académicocientífica en la que se están formando (Ezeiza, 2008; Álvarez, 2012), ocupan un lugar cada vez más relevante en las nuevas titulaciones. Entre estas, tiene un lugar destacado la competencia escritora presente ya en muchos currículos tanto de grado, como de postgrado y doctorado (Castelló, 2015).

En este marco, son cada vez más los casos en que se orienta la enseñanza a la producción de los géneros académicos con los que el alumno entra en contacto en los sucesivos niveles de su formación universitaria. Sin embargo, disponemos de poca investigación empírica sobre experiencias en las que se enseña a escribir textos académicos y científicos (Corcelles, Cano, Faz, y Vega, 2013) y, en consecuencia, tenemos poco conocimiento sobre los diversos tipos de retos a los que se tiene que ir enfrentando el estudiante universitario cuando ha de escribir tales textos.

Este artículo se plantea caracterizar los desafíos que se identifican en la elaboración del género ponencia por parte de un grupo de doctorandos. Estos participan en una experiencia docente que tiene como finalidad fortalecer la escritura académica y, en concreto, la producción del género discursivo ponencia como una preparación adecuada para facilitar la socialización disciplinar, es decir, para facilitar la adquisición de conocimientos, estrategias y convenciones para comunicarse dentro de una comunidad discursiva y legitimar el conocimiento que se construye (Arnoux, 2006).

En esta ocasión, se centra la atención en dos de los segmentos de este género: la introducción y las conclusiones. En concreto, se plantean los objetivos siguientes:

- Identificar los movimientos discursivos que se siguen en la elaboración de la introducción y la conclusión de una ponencia.

- Analizar de qué manera las revisiones, de compañeros y directores, inciden en la reformulación de las categorías de contenido y en cómo éstas se organizan.

El artículo contiene, en primer lugar, el marco teórico referencial en el que se sustenta la investigación. El siguiente apartado, el marco empírico; las características de los informantes y el origen del corpus de análi- 
La construcción de la introducción y la conclusión del género "ponencia" en el marco de un taller de escritura académica

Manuela Álvarez Álvarez, Erlantz Velasco luzuriaga y Mari Mar Boillos Pereira

sis; se reseña, además, la intervención didáctica realizada y se presenta el procedimiento y las categorías de análisis. Finalmente, se muestran los resultados y las conclusiones del trabajo.

\section{Marco teórico}

Se consideran en este apartado los conceptos teóricos que sostienen el análisis de los datos y funcionan como el eje vertebrador de la práctica docente: el concepto de género ponencia y las secciones introducción y conclusiones, el enfoque teórico metodológico adoptado en el análisis y un acercamiento a la teoría sobre los procesos de reelaboración de textos en el proceso de construcción del conocimiento.

\section{El género ponencia}

La ponencia constituye uno de los actos de comunicación con mayor presencia en el ámbito científico y académico; sin embargo, los estudios sobre este género son excepcionales (Day, 2005; Duo de Brottier, 2005; Perona, 2006). Es un medio privilegiado para la inserción gradual del estudiante en una comunidad discursiva específica; destaca por su flexibilidad temática y posibilita elaboraciones sucesivas de una misma materia semántica, dando lugar a la creación de otros textos de géneros adyacentes (Arnoux, 2006,p. 99).

Se podría definir este género como un acto de comunicación realizado por uno o varios agentes, destinado a ser expuesto ante un público experto y con una finalidad muy concreta. El ponente, eminentemente, informa a unos oyentes ya iniciados en la temática y que tratan de actualizar unos conocimientos (Duo de Brottier, 2005).

Esta investigación se centra en dos de las secciones más relevantes dentro de la construcción de este género: la introducción y las conclusiones.

La sección introducción ha generado un gran número de investigaciones en diferentes géneros como artículos de investigación (Swales, 1981; Samraj, 2002; Acosta, 2006), trabajos finales de máster (Dudley-Evans, 1986; Samraj, 2008), tesis doctorales (Bunton, 2002; Gil Salom, Soler Monreal, y Carbonell Olivares, 2008) y otro tipo de géneros (Martínez, 2008). Representa un segmento fundamental en todos estos géneros y, 
también, en la ponencia; en la interacción entre el ponente y los oyentes al servir de guía para conocer la estructura y el contenido de la investigación a la que da lugar. En palabras de Bunton (2002), la introducción desempeña un papel fundamental en un gran número de géneros disciplinares ya que se trata de una sección cuya finalidad es presentar y demostrar la pertinencia de una investigación científico-académica. Bhatia (1993) añade que la sección de introducción es capaz de promocionar una investigación a través de estrategias de persuasión.

Por su parte, la sección de conclusiones ha sido uno de los segmentos que menor atención ha recibido por parte de la comunidad científica (Dudley-Evans, 1986; Hopkins y Dudley-Evans, 1988; Yang y Allison, 2003; Bunton, 2005). Todos estos estudios se han centrado en el análisis de las conclusiones en el género tesis y en los artículos de investigación. La inclusión de esta sección como foco de análisis en esta investigación se debe a su relevancia en la configuración del género ponencia ya que, en ella, el autor muestra de manera sintetizada los hallazgos de su investigación, y por ende la aportación que esta realiza en la generación de conocimiento.

\section{Modelo teórico-metodológico adoptado}

Esta investigación se apoya en la línea de trabajo iniciada por Swales (1981, 1990, 1994) y Bhatia (1993) del Análisis de Género. Estos autores consideran el género como herramienta para analizar el lenguaje tanto en contextos académicos, como en contextos profesionales. Desde esta perspectiva teórica, el género se caracteriza como un evento comunicativo, con propósitos sujetos a una comunidad discursiva y con unos patrones relativamente estables.

La metodología fundamentada por esta tradición swalesina se basa en un modelo de análisis de la retórica a nivel global o seccional, que a través de movimientos retóricos y pasos establece los propósitos comunicativos asociados a los diferentes fragmentos que componen el texto (Morales, 2011). Para el análisis de la sección de "introducción" se ha tomado el modelo de análisis CARS propuesto por Swales (1990), mientras que para el analisis de las conclusiones se ha seleccionado como instrumento de análisis la propuesta de Yang y Allison (2003). 
La construcción de la introducción y la conclusión del género "ponencia" en el marco de un taller de escritura académica

Manuela Álvarez Álvarez, Erlantz Velasco luzuriaga y Mari Mar Boillos Pereira

\section{Los procesos de reelaboración de textos en la construcción del conocimiento}

Otra de las cuestiones clave de esta investigación es la función que se otorga a las actividades de revisión a la hora de proyectar la enseñanza de la escritura de textos académicos, caracterizados precisamente por no ser el resultado de una escritura lineal, sino por atravesar por distintas versiones provisionales. Tanto la planificación y revisión individual, como la revisión colaborativa (Castelló, 2007, Vargas 2005) han revelado una significativa incidencia en la mejora de los procesos de producción de textos académicos. Diversas investigaciones referentes a la enseñanza de la escritura han puesto de manifiesto los beneficios que incorpora la revisión colaborativa a las versiones provisionales que construyen los estudiantes pero, como se apunta, "no todas las situaciones de revisión entre iguales resultan igualmente útiles cuando se trata de evaluar el impacto en la mejora de la calidad de los textos" (Corcelles, Cano, Bañales, y Alicia, 2013).

Los procesos de revisión individual implican importantes esfuerzos mentales para el escritor. Se trata de un ejercicio que permite perfeccionar los productos textuales mediante la modificación y reelaboración de aspectos que percibe como desajustes. Un ejercicio fundamental para que los textos que producen los estudiantes adquieran la necesaria efectividad y calidad comunicativa y elimine las irrelevancias e inconsistencias de sus producciones. Por su parte, la revisión entre pares es una herramienta de gran valor para evaluar los distintos aspectos del proceso de producción y para objetivar la mirada del escritor mediante la aportación de comentarios externos (Vargas, 2005). Estas prácticas de revisión por pares consisten en el intercambio de borradores entre compañeros antes de ser entregados al profesor. Estas experiencias dotan al estudiante de una mirada crítica y externa de su trabajo, que sobrepasa las limitaciones que pudieran existir en la revisión de uno mismo. Podrían considerarse como un proceso de evaluación donde compañeros de un nivel similar examinan la calidad o el valor del producto de otros compañeros (Topping, 1998), lo que permite relanzar el proceso de escritura del escritor (Bessonnat, 2000). 


\section{Marco Empírico}

En este apartado se indican los informantes y el origen del corpus de análisis; se reseña brevemente la intervención didáctica realizada y el procedimiento y las categorías de análisis.

\section{Los informantes}

Los informantes de esta investigación son seis doctorandos del programa de Educación de la Universidad de Deusto. Ellos produjeron durante su participación en el taller los textos que son objeto de estudio en este trabajo.

\section{Características de la intervención}

La intervención docente consistió en un taller de escritura académica organizado con la finalidad de contar con un espacio que, por una parte, permitiera presentar los elementos teóricos para reflexionar acerca del uso de la lengua en una situación de comunicación dada y facilitar la construcción de criterios que orienten la producción del texto que responda a ella; y, por otra parte, un espacio que propiciara la interacción colaborativa basada en las producciones personales. La asistencia fue totalmente voluntaria y fue seguida por los seis doctorandos participantes y la coordinadora del taller; también participan en la experiencia las directoras de los doctorandos.

Este taller, que se lleva a cabo entre octubre y marzo del curso académico 2012-2013, se desarrolla en torno a un proceso tutorizado de elaboración de una ponencia por parte de cada uno de los seis informantes. Se estructura en dos fases: 
La construcción de la introducción y la conclusión del género "ponencia" en el marco de un taller de escritura académica

Manuela Álvarez Álvarez, Erlantz Velasco luzuriaga y Mari Mar Boillos Pereira

Tabla 1. Estructura del taller de escritura académica.

\begin{tabular}{|c|c|c|c|}
\hline Fases & Fecha & Duración & Tarea \\
\hline Fase 1 & Octubre de 2012 & $10 \mathrm{~h}(2,5 \mathrm{~h})$ & $\begin{array}{l}\text { Aproximación teorica al género } \\
\text { ponencia }\end{array}$ \\
\hline \multirow[t]{2}{*}{ Fase 2} & Noviembre de 2012 & & $\begin{array}{l}\text { Selección de tema de la ponencia } \\
\text { con el director de tesis }\end{array}$ \\
\hline & Enero de 2013 & $2 \mathrm{~h}$ & $\begin{array}{l}\text { Redacción del primer borrador de } \\
\text { la ponencia } \\
\text { Se establecen los criterios para la } \\
\text { revisión de lectores intermedios } \\
\text { Redacción del segundo borrador de } \\
\text { la ponencia } \\
\text { Revisión de los directores } \\
\text { Sesión orientada a considerar el } \\
\text { impacto que la experiencia de ela- } \\
\text { boración tutorada y compartida }\end{array}$ \\
\hline
\end{tabular}

\section{El corpus de análisis y procedimiento de recogida de datos}

El corpus de análisis de este trabajo está formado por el conjunto de las diversas versiones de la ponencia y las aportaciones que la revisión por pares y la realizada por la directora de tesis de los informantes aporta.

\section{Procedimiento de análisis de los datos}

El análisis de los datos se realiza orientado por la respuesta a las siguientes preguntas de investigación:

- ¿Qué movimientos discursivos, pasos y subpasos se despliegan en la construcción de la introducción y conclusión a través de las sucesivas reelaboraciones?

- ¿Cómo afecta la revisión de los lectores intermedios en el proceso de construcción de la introducción y la conclusión?

Los investigadores analizan conjuntamente los dos borradores y el texto definitivo de la ponencia; los casos dudosos se discuten hasta llegar a un consenso en su valoración. 


\section{Resultados y discusión}

\section{Estructura de las introducciones}

\section{LOS MOVIMIENTOS DISCURSIVOS}

La primera cuestión que llama la atención es la existencia de comportamientos diferentes por parte de los informantes al iniciar la construcción de las introducciones de sus ponencias tal y como puede visualizarse en la Tabla 3.

Tabla 3. Secuencia de movimientos retóricos en los tres borradores.

\begin{tabular}{llll}
\hline Informante & \multicolumn{2}{c}{ Secuencia de movimientos } \\
\hline & Borrador 1 & Borrador 2 & Borrador 3 \\
\hline 1 & $\mathrm{M} 1$ & $\mathrm{M} 1-\mathrm{M} 3$ & $\mathrm{M} 1-\mathrm{M} 3$ \\
\hline 2 & $\mathrm{M} 1-\mathrm{M} 2$ & $\mathrm{M} 1-\mathrm{M} 2-\mathrm{M} 3$ & $\mathrm{M} 1-\mathrm{M} 2-\mathrm{M} 3$ \\
\hline 3 & $\mathrm{M} 3$ & $\mathrm{M} 1-\mathrm{M} 2-\mathrm{M} 3$ & $\mathrm{M} 1-\mathrm{M} 2-\mathrm{M} 3$ \\
\hline 4 & $\mathrm{M} 1-\mathrm{M} 2$ & $\mathrm{M} 1-\mathrm{M} 2$ & $\mathrm{M} 1-\mathrm{M} 2$ \\
\hline 5 & $\mathrm{M} 3$ & $\mathrm{M} 3$ & $\mathrm{M} 3$ \\
\hline 6 & $\mathrm{M} 1-[\mathrm{M} 3]$ & $\mathrm{M} 1-[\mathrm{M} 3]$ & $\mathrm{M} 1-[\mathrm{M} 3]$ \\
\hline
\end{tabular}

Nota: [ ] Indica un movimiento incrustado en otro movimiento

El análisis de los movimientos retóricos en el primer borrador manifiesta dos tendencias claras. Una, la de aquellos informantes que concentran mayor cantidad de información en cuestiones relativas al establecimiento del tema (M1), explicando y detallando el tema general en el que se sitúa la investigación, su importancia y las investigaciones previas. Estos casos, cuatro de los seis informantes $(1,2,4$ y 6$)$, comienzan el discurso de la introducción estableciendo la situación temática de la ponencia y representan la tendencia más proclive a hablar del tema de una forma general. Además, manifiestan dificultades para establecer y ocupar el nicho o campo de estudio.

1. "El estudio de los valores ha cobrado especial relevancia en los últimos años. El nuevo paradigma socioeconómico y cultural ha traído consigo una discusión social entorno a la existencia y transmisión de valores, lo cual ha repercutido en el sistema educativo y se ve reflejado en la legislación educativa actual, donde se incide en la responsabilidad social de la educación para fomentar en los adolescentes valores para promover un mundo más justo y tolerante". Informante 6 
La construcción de la introducción y la conclusión del género "ponencia" en el marco de un taller de escritura académica

Manuela Álvarez Álvarez, Erlantz Velasco luzuriaga y Mari Mar Boillos Pereira

Otra, la representada por los informantes 3 y 5 para quienes la introducción se concentra en su totalidad en presentar la investigación (movimiento discursivo 3) informando sobre sus objetivos, la metodología empleada y, además, en el caso del informante 3, la estructura del trabajo.

2. "En primer lugar, desde la perspectiva teórica con el objetivo de situar este estudio dentro del contexto de investigación que se ha desarrollado hasta el momento.[...] Por ello, los objetivos de este estudio son los siguientes:" Informante 5

De los tres movimientos discursivos, el M2 es el menos recurrente. Aparece únicamente en dos de los seis informantes (el 2 y el 4) a través de la indicación del problema por el que se genera el estudio o el vacío existente en el campo de investigación.

3. "Aun compartiendo estas características, en la práctica, los programas de formación para madres y padres son muy distintos, y sus diferencias están [...]". Informante 2

Tal y como señala Swales (1990), se trata de un movimiento que requiere un mayor nivel de concreción que el anterior. Los resultados que se obtienen en este estudio vienen a refrendar las investigaciones Ilevadas a cabo por otros autores (Gil-Salom, Soler-Monreal, y CarbonellOlivares, 2008) que señalan que el establecimiento del nicho es el menos recurrente también en otros géneros académicos como el caso de la Tesis Doctoral.

Finalmente, y con respecto al primer borrador, cabe destacar que ningún informante establece el modelo secuencial protótipico de M1-M3 establecido para escritos de tradición hispana (Gil-Salom, Soler-Monreal, y Carbonell-Olivares, 2008; Carbonell-Olivares, Gil-Salom, y SolerMonreal, 2009; Soler-Monreal, Carbonell-Olivares, y Gil-Salom, 2011) ni, por supuesto, el modelo secuencial prototípico de M1-M2-M3 de los textos escritos en lengua inglesa. Por su parte, el análisis del segundo borrador manifiesta un cambio sustancial en la construcción de las introducciones para la mitad de los informantes, en concreto, los informantes 1,2 y 3.

Se completa la introducción con el desarrollo del M3, aportando información relativa a los objetivos del estudio (caso del informante 1) o a los objetivos del estudio, cuestiones metodológicas y resultados previstos (informante 2). Para el informante 3 supone, sin embargo, el establecimiento de la temática y la identificación del vacío dentro del campo de estudio, así como una indicación de la necesidad por la que se 
investiga (movimientos 1 y 2). Con estos cambios se produce un mayor acercamiento al modelo prototípico M1-M2-M3, anteriormente mencionado. Dos de los seis informantes ( 2 y 3 ) introducen los 3 movimientos establecidos para la introducción.

4. "[...] nos proponemos analizar 15 programas para encontrar qué elementos tienen en común y cuáles son las recomendaciones de los diferentes autores, con el objetivo de elaborar una propuesta de elementos clave para la realización de programas de formación parental. Comenzaremos con la descripción de los programas analizados, así como con el método de investigación que se ha seguido, y a continuación se detallarán los resultados del análisis y por último las conclusiones, en las que se elabora la propuesta de puntos clave". Informante 2

Por otro lado, resulta interesante observar que el paso del primer al segundo borrador tiene una mayor incidencia en relación al M3: cinco de los seis informantes aportan información concreta sobre el estudio que van a realizar, igual número de informantes que en el caso del M1. Por el contrario, el M2 se muestra como el reto más difícil al que se enfrentan los doctorandos; tres de los seis lo desarrollan.

En lo que respecta al tercer y último borrador cabe destacar que los cambios con respecto al segundo borrador son de menor relevancia. En efecto, 5 de los 6 informantes $(1,2,4,5$ y 6$)$ no realizan ningún cambio y, en el caso del informante 3 , se advierte únicamente un mayor desarrollo informativo en el M1 con la incorporación de datos relativos a investigaciones previas. En el caso del M2 se incorpora una indicación de la necesidad de estudio en dicho campo:

5. "Con estas consideraciones, el hecho de poder realizar una contribución empírica para validar una metodología mediante la que determinar el grado en que el alumnado consigue adquirir la "Competencia para la autonomía e iniciativa personal" suscitaba para nosotros gran interés, ya que en tanto en cuanto ésta sea evaluada de forma sistemática, se podrá obtener evidencia del proceso de enseñanza y aprendizaje de la misma y su esperable beneficio". Informante 2

\section{PASOS Y SUBPASOS DE LOS MOVIMIENTOS DISCURSIVOS}

El análisis de los pasos y subpasos que se despliegan en los tres movimientos discursivos viene a apoyar los resultados anteriormente señalados. Es 
La construcción de la introducción y la conclusión del género "ponencia" en el marco de un taller de escritura académica

Manuela Álvarez Álvarez, Erlantz Velasco luzuriaga y Mari Mar Boillos Pereira

el establecimiento de la temática el movimiento discursivo (M1) el que se desarrolla a través de una proporción mayor de pasos y subpasos.

Tabla 4. Pasos y subpasos encontrados del M1- establecimiento de la temática en los informantes.

\begin{tabular}{lcccc}
\hline Pasos & $\begin{array}{c}\text { Borra- } \\
\text { dor } 1\end{array}$ & $\begin{array}{c}\text { Borrador } \\
2\end{array}$ & $\begin{array}{c}\text { Borrador } \\
3\end{array}$ & $\begin{array}{c}\text { Número } \\
\text { total de } \\
\text { casos }\end{array}$ \\
\hline M1P1. Importancia del tema & $1,4,6$ & $1,3,4,6$ & $1,3,4,6$ & 4 \\
\hline $\begin{array}{l}\text { M1P2: Generalización y antecedentes } \\
\text { del tema }\end{array}$ & & & & \\
\hline $\begin{array}{l}\text { M1SP2A: Situar el problema o nece- } \\
\text { sidad }\end{array}$ & 2,6 & 2,6 & 2,6 & 2 \\
\hline $\begin{array}{llll}\text { M1SP2B: Indicación de limitaciones } \\
\text { M1SP2C: Ejemplificaciones }\end{array}$ & & & & \\
\hline $\begin{array}{l}\text { M1SP2D: Definición de términos } \\
\text { (clasificación y comentarios termino- } \\
\text { lógicos) }\end{array}$ & 2,4 & 2,4 & 2,4 & 2 \\
\hline M1SP2E: Propuesta de soluciones & & & \\
\hline $\begin{array}{l}\text { M1P3: Definición de términos (clasi- } \\
\text { ficación) }\end{array}$ & & & & \\
\hline $\begin{array}{l}\text { M1P4: Revisión de investigaciones } \\
\text { previas }\end{array}$ & $1,2,6$ & $1,2,6$ & $1,2,6$ & 3 \\
\hline $\begin{array}{l}\text { M1P5: Referencia al contexto institu- } \\
\text { cional o equipo de investigación }\end{array}$ & & & \\
\hline
\end{tabular}

Fuente: Adaptado de Carbonell-Olivares, M., Gil-Salom, L., y Soler-Monreal, C. (2009). The schematic structure of Spanish PhD thesis introductions. Spanish in Context, 6 (2), 151-175.

Como se visualiza en la Tabla 3, los pasos de mayor frecuencia de aparición son el de importancia del tema (P1) y el de revisión de investigaciones previas (P4). El primero se encuentra en 4 de los 6 informantes, mientras que el otro se identifica en 3.

El paso de generalización y antecedentes del tema (P2) se halla diversificado en dos subpasos: situar el problema (A) y definición de términos (D):

6. "Los valores resultan un eje fundamental para el pleno desarrollo de los individuos como personas responsables y activas dentro de la sociedad en la que viven (...)" P2A. Informante 6

7. “(...) partiendo de las definiciones sobre Educación Inclusiva que se exponen (...)"Informante 4 
En lo que respecta al movimiento 3 cabe señalar, también, la existencia de una alta recurrencia de pasos y subpasos.

Tabla 5. Pasos y subpasos encontrados del M3- ocupación del nicho en los informantes.

\begin{tabular}{|c|c|c|c|c|}
\hline \multirow[t]{2}{*}{ Pasos } & \multicolumn{3}{|c|}{ Informante } & \multirow{2}{*}{$\begin{array}{l}\text { Número } \\
\text { total de } \\
\text { casos }\end{array}$} \\
\hline & $\begin{array}{l}\text { Borra- } \\
\text { dor } 1\end{array}$ & $\begin{array}{l}\text { Borra- } \\
\text { dor } 2\end{array}$ & $\begin{array}{l}\text { Borra- } \\
\text { dor } 3\end{array}$ & \\
\hline M3P1: Propósitos y objetivos & 3,5 & $1,3,5$ & $\begin{array}{l}1,2 \\
3,5\end{array}$ & 4 \\
\hline \multicolumn{5}{|l|}{$\begin{array}{l}\text { M3P2: Presentación y anuncio del trabajo } \\
\text { de investigación } \\
\text { M3SP2A: Trabajo realizado } \\
\text { M3SP2B: Enfoque de la investigación } \\
\text { M2SP2C: Limitaciones del estudio } \\
\text { M3SP2D: Requisitos previos }\end{array}$} \\
\hline \multicolumn{5}{|l|}{ M3P3: Campo de estudio } \\
\hline $\begin{array}{l}\text { M3P4: Metodología (Parámetros de la } \\
\text { investigación) }\end{array}$ & 3,5 & $3,5 \quad 2$ & $2,3,5$ & 3 \\
\hline M3P5: Instrumentos & & & 2 & 1 \\
\hline $\begin{array}{l}\text { M3P6: Resultados (Anuncio de los prin- } \\
\text { cipales hitos o presentando el producto o } \\
\text { modelo propuesto) }\end{array}$ & & & 2 & 1 \\
\hline \multicolumn{5}{|l|}{ M3P7: Justificación } \\
\hline \multicolumn{5}{|l|}{$\begin{array}{l}\text { M3P8: Estructura de la ponencia } \\
\text { M3P8A:Estructura global de la ponencia } \\
\text { M3P8B: Estructura de los apartados de la } \\
\text { ponencia } \\
\text { M3P8C: Contenidos de los apartados de la } \\
\text { ponencia } \\
\text { M3P8D: Objetivos de los apartados de la } \\
\text { ponencia }\end{array}$} \\
\hline \multicolumn{5}{|l|}{ M3P9: Hipótesis } \\
\hline \multicolumn{5}{|l|}{$\begin{array}{l}\text { M3P10: Aplicabilidad de los resultados de } \\
\text { la tesis }\end{array}$} \\
\hline $\begin{array}{l}\text { M3P11: Evaluación de los resultados de la } \\
\text { tesis }\end{array}$ & & & & \\
\hline
\end{tabular}

Fuente: Adaptado de Carbonell-Olivares, M., Gil-Salom, L., y Soler-Monreal, C. (2009). The schematic structure of Spanish PhD thesis introductions. Spanish in Context, 6 (2), 151-175. 
Ahora bien, en el caso concreto del M3, y a diferencia de lo que se veía para el M1, el despliegue de los pasos y los subpasos es el resultado de las diversas reescrituras y afecta a menor número de informantes. Los pasos más recurrentes se representan a través de la exposición de los objetivos (P1) y la metodología (P4). El primero de ellos (P1) a través de los informantes 1, 2, 3 y 5 y en el segundo caso (P4), presente en los informantes 2,3 y 5 .

8. "La segunda fase de este trabajo afronta el análisis cualitativo de textos descriptivos de la totalidad de estudiantes arabófonos de un curso de inmersión [...] Por ello, los objetivos de este estudio son los siguientes:[...]" Informante 5

En cambio, como se ha observado en el ejemplo 4, los pasos de instrumentos (P5) y anuncio de los resultados (P6) solo se constatan en un informante cada uno.

En lo que respecta a los pasos y subpasos identificados para el movimiento de establecimiento del nicho (M2) la situación es bien distinta como puede verse en la siguiente tabla.

Tabla 5. Pasos y subpasos encontrados del M2- establecimiento del nicho en los informantes.

\begin{tabular}{lcccc}
\hline Pasos & & Informante & $\begin{array}{c}\text { Número } \\
\text { total de } \\
\text { casos }\end{array}$ \\
\hline & $\begin{array}{c}\text { Borra- } \\
\text { dor 1 }\end{array}$ & $\begin{array}{c}\text { Borra- } \\
\text { dor 2 }\end{array}$ & $\begin{array}{c}\text { Borra- } \\
\text { dor 3 }\end{array}$ & \\
\hline $\begin{array}{l}\text { M2P1A: Indicación del vacío en la investi- } \\
\text { gación }\end{array}$ & 2 & 2,3 & 2,3 & 2 \\
\hline $\begin{array}{l}\text { M2P1B: Indicación del problema o necesi- } \\
\text { dad }\end{array}$ & 2,4 & 2,4 & 2,4 & 2 \\
\hline M2P1C: Preguntas de investigación & & 4 & 4 & 1 \\
\hline $\begin{array}{l}\text { M2P1D: Continuación de la línea de inves- } \\
\text { tigación }\end{array}$ & & & & \\
\hline
\end{tabular}

Fuente: Adaptado de Carbonell-Olivares, M., Gil-Salom, L., y Soler-Monreal, C. (2009). The schematic structure of Spanish PhD thesis introductions. Spanish in Context, 6 (2), 151-175.

Se hallan 3 de los 4 pasos: la identificación del vacío y el problema (1A) o la necesidad de la investigación (1B) y preguntas de investigación (1C); y su presencia se limita a dos informantes. 
9. "[...] también queda de manifiesto que [...] aún queda un camino por recorrer". Informante 3

\section{Estructura de las conclusiones}

La elaboración de las conclusiones responde a un patrón de construcción más homogéneo. Todos los informantes se centran en el desarrollo del M1 (resumen de resultados), siendo éste en extensión, el movimiento preponderante. Además, la mayoría de ellos (1, 2, 4 y 5$)$ da inicio a las conclusiones con este movimiento y dos de ellos (1 y 2) únicamente desarrollan este movimiento en toda la sección.

Tabla 6. Secuencia de movimientos retóricos en el los 3 borradores.

\begin{tabular}{llll}
\hline Informante & \multicolumn{4}{l}{ Secuencia de movimientos } \\
\hline & Borrador 1 & Borrador 2 & Borrador 3 \\
\hline 1 & $\mathrm{M} 1$ & $\mathrm{M} 1-\mathrm{M} 3$ & $\mathrm{M} 1-\mathrm{M} 3$ \\
\hline 2 & $\mathrm{M} 1$ & $\mathrm{M} 1$ & $\mathrm{M} 1$ \\
\hline 3 & $\mathrm{M} 2-\mathrm{M} 1-\mathrm{M} 2$ & $\mathrm{M} 2-\mathrm{M} 1-\mathrm{M} 2$ & $\mathrm{M} 2-\mathrm{M} 1-\mathrm{M} 2-\mathrm{M} 3$ \\
\hline 4 & $\mathrm{M} 1-\mathrm{M} 2$ & $\mathrm{M} 1-\mathrm{M} 2$ & $\mathrm{M} 1-\mathrm{M} 2$ \\
\hline 5 & $\mathrm{M} 1-\mathrm{M} 2-\mathrm{M} 3$ & $\mathrm{M} 1-\mathrm{M} 2-\mathrm{M} 3$ & $\mathrm{M} 1-\mathrm{M} 2-\mathrm{M} 3$ \\
\hline 6 & $\mathrm{M} 2-\mathrm{M} 1$ & $\mathrm{M} 2-\mathrm{M} 1$ & $\mathrm{M} 2-\mathrm{M} 1$ \\
\hline
\end{tabular}

Nota: [ ] Indica un movimiento incrustado en otro movimiento

Como se aprecia en la Tabla 6, no se producen cambios reseñables a excepción de la inclusión del movimiento 3 en la reelaboración segunda del informante 1 y en la reelaboración tercera del informante 3.

Por otra parte, en el primer borrador, y en lo que al M1 o resumen de resultados se refiere, cabe destacar que este se desarrolla de manera similar entre los informantes 1, 2, 4, 5 y 6 , en los que se recogen los resultados de la propia investigación de manera concisa y concreta.

10. "A nivel de objetivos: [...] Como podemos observar, existe una clara relación entre los objetivos de los programas de formación de padres y madres y las necesidades familiares percibidas...". "A nivel de contenidos: [...]. Los contenidos que más se suelen utilizar en el trabajo de estas áreas son: por un lado, los sentimientos percibidos por los padres y las madres en torno a su tarea parental, y el manejo de emociones negativas relacionadas con la frustra- 
La construcción de la introducción y la conclusión del género "ponencia" en el marco de un taller de escritura académica

Manuela Álvarez Álvarez, Erlantz Velasco luzuriaga y Mari Mar Boillos Pereira

ción..." Informante 2

11. "A través de los resultados obtenidos en el siguiente estudio, existen diversas conclusiones que pueden extraerse. [...] Por un lado, desde el punto de vista teórico, ha quedado de relieve el vacío existente en el ámbito de investigación de la escritura en español por arabófonos en la actualidad." Informante 5

Por el contrario, en el caso del informante 3 no se resuelve el desarrollo de este movimiento según lo esperado. Más que presentar una síntesis de los resultados, lo que hace es indicar las fases de la investigación.

12. "Con la realización de las 2 fases de la investigación se dio respuesta a los 3 objetivos propuestos: Identificación de necesidades de formación del profesorado sobre evaluación, diseño del modelo para evaluar la competencia y validación del mismo. Informante 3

Por su parte el M2, evaluación de los resultados, se encuentra en 4 de los 6 informantes $(3,4,5$ y 6$)$.

13. "De la valoración final del plan realizada por el grupo de discusión, se desprende que la puesta en práctica de dicho modelo, supuso un esfuerzo de adaptación a una metodología fundamentada en el enfoque competencial, pero a la vez, sirvió para dotar al centro de herramientas y estrategias útiles para evaluarla" Informante 3

El M3 solo se encuentra en el primer borrador del informante 5:

14. "Por el contrario, el fin último de este estudio es tomar unos casos concretos de un contexto de aula para hacer una descripción de la realidad y comprobar si aquellos métodos que se plantean son válidos para poder llevar a cabo estudios de mayor envergadura y con un número superior de informantes". Informante 5

Los cambios que se producen respecto ese primer borrador afectan, precisamente, a este último movimiento. El informante 1 introduce información relativa a las conclusiones derivadas de la investigación (M3) en la segunda y tercera reelaboración y, por su parte, el informante 3, la introduce también, pero en la reelaboración tercera. Ejemplos:

15. "En este sentido, este artículo, como parte de un estudio más amplio sobre la movilidad académica, quiere poner de relieve de cara a futuras investigaciones, la necesidad de definir y tener en cuenta los distintos acercamientos a éste fenómeno.". Informante 1 
16. "Teniendo en cuenta los resultados obtenidos, consideramos conveniente seguir desarrollando el modelo de evaluación, ampliándolo a la evaluación de las competencias "para aprender a aprender" y "Social y ciudadana" en educación infantil y primaria. Informante 3

\section{Incidencia de los lectores intermedios en la construcción de la introducción y la conclusión de las ponencias}

El análisis de las propuestas de cambio realizadas por los lectores intermedios, compañeros y directoras, no parece tener la incidencia que cabría esperar en lo que a la construcción discursiva de las introducciones y las conclusiones se refiere.

En la introducción la revisión por pares afecta solamente a los informantes 1 y 2 a los que se les sugiere incluir información acerca de los objetivos y estructura del trabajo; es decir, información asociada al tercero de los movimientos retóricos: la ocupación del nicho. La intervención de las directoras tampoco aporta sugerencias vinculadas al desarrollo de los movimientos retóricos; únicamente se indica al informante 3 la necesidad de incluir la subcategoría de resultados, asociada al M3.

En el caso de las conclusiones, la situación es muy similar. La revisión por pares sólo tiene incidencia en aspectos de la elaboración del texto de carácter local, a excepción de los casos de los informantes 1 y 6 quienes reciben sugerencias que modifican la estructura retórica. En el primer caso, se sugiere incluir información relativa a la significación de la investigación, es decir, el paso 1 recogido en el movimiento 2 (evaluación de resultados) y, en el segundo, la incorporación de información relativa a la propuesta metodológica.

\section{Conclusiones}

De los resultados anteriores se desprenden una serie de conclusiones que pasamos a presentar a continuación:

- El patrón de construcción discursiva es más homogéneo en las conclusiones que en las introducciones. Todos los informantes tienen conciencia de que el segmento conclusión despliega un 
resumen o una recapitulación de los resultados del trabajo y casi todos, además, desarrollan una valoración de los mismos. E incluso, la mitad de los informantes incluye el M3, si bien en este caso tal ensanchamiento es fruto de las aportaciones de los lectores intermedios. Por el contrario, en el caso de la construcción inicial de las introducciones se manifiestan dos tendencias aunque la predominante sea concentrar la mayor cantidad de información en cuestiones relativas al establecimiento del tema (M1). Además, ese patrón inicial sufre cambios; se desarrolla un proceso de reelaboración más largo que va, como tendencia, desde lo más abstracto (M1) hacia la concreción del trabajo que se va a realizar (M3) pasando de soslayo, en algunos casos, por el M2. Y esto en el caso concreto de unos informantes del ámbito de las ciencias sociales y humanas.

- Los resultados del análisis de los pasos y subpasos de los movimientos discursivos en los que se desenvuelven las introducciones no hacen sino confirmar las anteriores conclusiones. El establecimiento de la temática se desarrolla a través de una proporción mayor de pasos y subpasos que en el caso de la presentación de la investigación y, además, los que se desarrollan son el resultado de varias reescrituras y conciernen a un número menor de informantes. No obstante, es necesario matizar que el despliegue informativo sobre cómo se ocupa el espacio atiende a un menor número de pasos y subpasos de los señalados para textos académicos como el artículo de investigación. El caso de la ponencia es un texto académico que participa de una construcción discursiva similar, pero al que se le suele conceder una menor extensión, luego sería también esperable un desarrollo menor que podría justificar la presencia de menos pasos.

- Tanto en el caso de las introducciones como en el de las conclusiones, los textos definitivos no desarrollan los movimientos discursivos que cabría esperar en la elaboración un texto académico como es la ponencia por parte de universitarios que se sitúan en la trayectoria final de su formación como es la del doctorado.

- Las diferentes reescrituras no han conducido a que las introducciones respondan al esquema clásico de M1-M2-M3 más que en la mitad de los casos analizados. 
Si bien el estudio tiene una importante limitación en cuento a que son pocos los informantes participantes, sus resultados parecen tener implicaciones claras en la práctica docente. Entre éstas, cabe destacar,

- La necesidad ineludible de que las prácticas en el aula respondan a actividades comunicativas contextualizadas de manera que la escritura esté orientada a quien se dirige la comunicación y no centrada en el tema del que se habla.

- La conveniencia de que las prácticas de escritura incidan en la reflexión acerca de cómo se va construyendo la información de un texto. Esta reflexión debe ir más allá de la aplicación de una estructura entendida como un molde que se repite de manera sistemática y mecánica en la confección de cualquier texto.

- La importancia de que la actividad de revisión por pares venga precedida de una preparación para que ésta alcance la eficacia que se espera.

\section{Referencias bibliográficas}

Acosta, O. (2006). Análisis de introducciones de artículos de investigación publicados en la Revista Núcleo 1985-2003. Núcleo, 18 (23), 9-30.

Álvarez, M. (2012). La relevancia de las competencias comunicativas en la formación del estudiante universitario y retos que plantea. En M. Acillona, Marcos interpretativos de la realidad social contemporánea (pp. 29-41). Bilbao: Publicaciones de la Universidad de Deusto.

Arnoux, E. (2006). Incidencia de la lectura de pares y expertos en la reescritura de tramos del trabajo de tesis. RLA: Revista de lingüística teórica y aplicada, (44), 95-118.

Bessonnat, D. (2000). Deux ou trois choses que je sais de la réécriture. Pratiques, 105$106,5-22$.

Bhatia, V. (1993). Analysing genre: Language use in professional setting. Nueva York: Longman.

Bunton, D. (2002). Generic moves in PH. D thesis introductions. En J. Flowerdew, Academic discourse (pp. 57-75). Essex: Pearson Education.

Bunton, D. (2005). The structure of PhD conclusion chapters. Journal of English for Academic Purposes, (4), 207-224.

Carbonell-Olivares, M., Gil-Salom, L., y Soler-Monreal, C. (2009). The schematic structure of Spanish PhD thesis introductions. Spanish in Context, 6 (2), 151-175.

Castelló, M. (2007). Escribir y comunicarse en contextos científicos y académicos. Barcelona: Graó.

Castelló, M. (2015). Los retos actuales de la alfabetización académica: estado de la cuestión y últimas investigaciones. Enunciación, 19 (2), 346-365. 
La construcción de la introducción y la conclusión del género "ponencia" en el marco de un taller de escritura académica

Manuela Álvarez Álvarez, Erlantz Velasco luzuriaga y Mari Mar Boillos Pereira

Corcelles, M., Cano, M., Bañales, G., y Alicia, N. (2013). Enseñar a escribir textos científico-académicos mediante la revisión colaborativa: El trabajo final de grado en Psicología. REDU: Revista de docencia universitaria, 11 (1), 79-100.

Day, R. (2005). Cómo escribir y publicar trabajos científicos. Washington: Pan American Health Org.

Dudley-Evans, T. (1986). Genre anlysis: an investigation of the introduction and discussion sections of MSc dissestrations. Talking about text, 128-145.

Duo de Brottier, O. (2005). La ponencia y el resumen de ponencia. En L. Cubo de Severino, Los textos de la ciencia (pp. 113-150). Córdoba: Comunicarte.

Ezeiza, J. (2008). Las competencias comunicativo-lingüísticas de tipo disciplinar: perfiles profesionales y recursos para su desarrollo en el nuevo EEES. Actas del XXXVII Simposio Internacional de la Sociedad Española de Lingüística, (pp. 131-144).

Gil-Salom, L., Soler-Monreal, C., y Carbonell-Olivares, M. (2008). The move-step structure of the introductory sections of spanish phd theses. RESLA, (21), 85-106.

Hopkins, A., y Dudley-Evans, T. (1988). A genre-based investigation of the discussion sections in articles and dissertations. English for Specific Purposes, 7 (2), 113-122.

Martínez, C. (2008). Estructura retórica en las introducciones de textos económicos. In 25 años de lingüística en España [Recurso electrónico]: hitos y retos= 25 years os applied linguistics in Spain: milestones and challenges, 479-487.

Perona, E. (2006). Lineamientos generales para escribir un paper o trabajo de investigación. Córdoba: Departamento de Economía y Finanzas, Facultad de Ciencias Económicas, Universidad Nacional de Córdoba.

Samraj, B. (2002). Introductions in research articles: Variations across disciplines. English for Specific Purposes, 21(1), 1-17.

Samraj, B. (2008). A discourse analysis of master's theses across disciplines with focus on introduction. English for Academic Purposes, (7), 55-67.

Soler-Monreal, C., Carbonell-Olivares, M., y Gil-Salom, L. (2011). A contrastive study of the rhetorical organisation of English and Spanish PhD thesis introductions. English for Specific Purposes, 30 (1), 4-17.

Swales, J. (1981). Aspects of article introductions. Birmingham: The university of Aston.

Swales, J. (1990). Genre Analysis: English in academic and research settings. Cambridge: University of Cambridge.

Swales, J., y Feak, C. (1994). Academic writing for graduate students. Ann Arbor: University of Michigan Press.

Swales, J. (2004). Research genres: explorations and applications. Cambridge: University of Cambridge.

Topping, K. (1998). Peer Assessment between Students in Colleges and Universities. Review of Educational Research, $68,249-276$.

Vargas, A. (2005). Escribir en la Universidad: reflexiones sobre el proceso de composición escrita en textos académicos. Lenguaje, 33, 97-125.

Yang, R., y Allison, D. (2003). Research articles in applied linguistics: Moving form results to conclusions. English for Specific Purposes, (22), 365-385. 